\title{
Value of multidetector computed tomographic angiography in marginally resectable pancreatic cancer
}

This article was published in the following Dove Press journal:

Reports in Medical Imaging

27 January 2015

Number of times this article has been viewed

\section{Dimitris Fagkrezos' \\ Christos Dervenis ${ }^{2}$ Charikleia Triantopoulou'}

'Radiology Department, ${ }^{2}$ Department of Surgery, Konstantopouleio Hospital Neas Ionias, Athens, Greece
Correspondence: Charikleia Triantopoulou Konstantopouleio General Hospital, Radiology Department, 3-5 Agias Olgas Street, 14233 N.IONIA,Athens, Greece Email ctriantopoulou@gmail.com
Background: Pancreatic carcinoma is a common gastrointestinal malignancy. Accurate preoperative imaging helps to avoid unnecessary or unsuccessful surgical procedures and reduce the number of aborted pancreatic resections. The purpose of this review is to determine the role of multidetector computed tomographic angiography (MDCTA), which allows rapid anatomic evaluation, in accurate local and distant staging of marginally resectable pancreatic adenocarcinomas.

Methods: Our methodology was based on the systematic search of published papers using PubMed/MEDLINE, Embase, and the Cochrane Central Register of Controlled Trials for the years 2009-2014, reporting on the reliability and diagnostic accuracy of MDCTA in the evaluation of pancreatic cancer resectability.

Results: Based on 33 articles analyzed in the review, MDCTA is the most widely available imaging modality for diagnosing and staging patients with pancreatic cancer, and it can be used for the surgical planning, specifically in locally advanced marginally resectable tumors.

Conclusion: Accurate pretreatment assessment of resectability is crucial to design appropriate preoperative protocols of the operating team. It is possible to achieve a complete resection at the first instance in some patients who have borderline resectable tumors as predicted on preoperative imaging. Preoperative MDCTA-based grading systems can help identify such patients. This would help to avoid delayed curative resections in such patients and minimize the risk of aborted open-close operations.

Keywords: MDCTA, MDCT angiography, borderline resectable tumors

\section{Introduction}

Pancreatic cancer is a lethal tumor presenting as a locally advanced or metastatic cancer in many patients. It is estimated that only about $20 \%-25 \%$ of patients have a potentially resectable cancer. Pancreatic adenocarcinoma is the fourth most common cause of death from cancer in the United States. The survival rate at 5 years is less than $5 \% ., 2$ About $60 \%$ of tumors are located in the head of the pancreas, $15 \%$ in the body, $5 \%$ in the tail, while $20 \%$ are diffuse within the pancreas. ${ }^{3}$ Multifocal tumors have also been reported. Pancreatic head tumors are usually smaller compared with those in the body and tail because of earlier symptoms due to the close contiguity with the bile duct. Imaging of pancreatic carcinoma has a crucial role in treatment decisions.

Surgical resection is the only potential curative treatment of pancreatic adenocarcinoma. Unfortunately, at surgery, only 5\%-30\% of tumors can be totally resected as they are usually more extended than predicted by imaging. ${ }^{4,5}$ Even in high volume centers, the Whipple procedure is related to a mortality of up to $4 \%$ while exploratory 
laparotomy shows a morbidity that can reach $25 \% .^{6}$ Therefore, it is important to identify all potentially resectable tumors and to avoid surgical procedures in patients with locally advanced/ unresectable disease. Increasingly accepted in pancreatic cancer surgery is the R classification to define the extent of the resection. $\mathrm{R} 0$ resection is the resection in which there is no microscopic evidence of tumor at any of the edges of the resection specimen. $\mathrm{R} 1$ resection is the resection in which tumor cells may be observed by microscopy at one or more edges of the resected specimen, in other words the complete resection with positive resection limits. Finally, $\mathrm{R} 2$ resection is a partial resection of a macroscopically visible tumor. It is proven that patients who finally undergo a margin positive resection ( $\mathrm{R} 1$ or R2) show similar survival as those with locally advanced disease; therefore, surgery should be avoided in these cases..$^{7-10}$ Thus, the clear determination of the resectability status at the time of initial staging is of great importance. This can be done by a computed tomography (CT) scan using a dedicated pancreatic protocol. ${ }^{11}$ Based on this high quality CT imaging and specific criteria used, pancreatic tumors are classified as resectable, locally advanced, or metastatic. Recently, a new category was recognized as "borderline resectable tumors (BRTs)," giving rise to conflicting statements on the accurate definition. ${ }^{12,13}$ It seems that these patients are not good candidates for surgery while the use of preoperative chemotherapy can ensure an $\mathrm{R} 0$ resection. Increasingly accepted in pancreatic cancer surgery is the $\mathrm{R}$ classification to define the extent of the resection. $\mathrm{R} 0$ resection is the resection in which there is no microscopic evidence of tumor at any of the edges of the resection specimen. R1 resection is the resection in which tumor cells may be observed by microscopy at one or more edges of the resected specimen; in other words, the complete resection with positive resection limits. Finally, $\mathrm{R} 2$ resection is a partial resection of a macroscopically visible tumor. Multidetector computed tomography (MDCT) is now the method of choice to estimate local tumor resectability, although small hepatic or peritoneal metastases could occasionally be missed. ${ }^{14-16}$ The application of multidetector computed tomographic angiography (MDCTA), that includes contrast-enhanced techniques, multiplanar reconstructions (MPR), and maximal-intensity projection (MIP) postprocessing, has led to an increased potential to identify and stage accurately the tumor, especially concerning vascular infiltration, with a reported accuracy for resectability of about $90 \% .{ }^{17-19}$

\section{Methods}

Our methodology was based on the systematic search of the published papers using PubMed/MEDLINE, Embase, and the Cochrane Central Register of Controlled Trials for the years 2009-2014, reporting on the reliability and diagnostic accuracy of MDCTA in the evaluation of pancreatic cancer resectability. ${ }^{20}$

The search terms that we used were "pancreatic cancer," "diagnosis," "staging," "resectability," "computed tomography," "MDCTA," "randomized controlled trials," "review," and "meta-analysis."

Articles on the role of MDCT scan in the diagnosis, local staging, and determination of resectability of pancreatic and periampullary adenocarcinoma as well as original papers on the added value of MDCTA were included. Case reports and articles on tumors other than pancreatic adenocarcinoma were excluded.

It should be clarified that the term staging indicated the ability of the imaging modalities to assess the tumor, the lymph nodes, and the metastases. On the other hand, the term resectability indicates the technical requirements concerning mainly vascular involvement.

\section{Results}

\section{Accuracy of MDCT}

There is no question that in the last years there has been a tremendous advance in CT scanners and techniques, and this had a great impact on improved resolution and diagnostic capability. Thin-cut (at least 64 section) intravenous contrast-enhanced MDCT is the imaging technique of choice for the investigation of pancreatic cancer. ${ }^{21}$ Pancreatic parenchymal and portal venous phases should be performed while arterial phase is optional. ${ }^{17}$

The sensitivity of CT in the detection of pancreatic tumors has improved in recent times and lies between $75 \%$ and $100 \%$ in different series, with a specificity of $70 \%-100 \% .^{22-29}$ However, the smaller the tumor is, the less the CT sensitivity. It is reported that $\mathrm{CT}$ sensitivity for lesions measuring $\leq 2 \mathrm{~cm}$ is between $68 \%$ and $77 \%{ }^{23,25}$ and the accuracy close to $77 \%{ }^{28}$ In these lesions, endoscopic ultrasound seems to perform better. ${ }^{28}$

It is important to know what contrast phase to use in addressing the clinical question. The pancreatic parenchymal and portal venous phases appear to be similar and better than the early arterial phase for delineating pancreatic adenocarcinomas. ${ }^{30,31}$ However, images obtained in the pancreatic phase suffer from more flow artifacts and decreased attenuation in the superior mesenteric vein (SMV) compared with the artifacts on images obtained in the portal venous (hepatic) phase. Therefore, for evaluating vascular invasion, images obtained in the portal venous phase are better. ${ }^{32}$

The issue of selecting the better contrast phase is still debated. Imbriaco et $\mathrm{al}^{33}$ showed that thin-section, single-phase MDCT is very accurate for the diagnosis and 
assessment of resectability in patients with a suspected pancreatic tumor due to the optimal tumor-to-pancreas contrast and maximal pancreatic parenchymal and peripancreatic vascular enhancement. On the other hand, portal phase allows visualization of the entire liver and the whole upper abdomen, revealing possible metastases and/or peritoneal seeding.

\section{Definitions of resectability}

The American Joint Committee on Cancer (AJCC) TNM (tumor, nodes, metastasis) staging for pancreatic cancer was revised in 2010 ( 7 th edition). In this edition, the important role of high quality CT imaging was emphasized while the resectability criteria used did not change in the latest AJCC edition. ${ }^{34}$ Criteria for resectability include the absence of distant metastases, the absence of tumor extension to the celiac artery and superior mesenteric artery (SMA) with clear fat planes around the vessels, and no imaging evidence of SMV or portal vein (PV) distortion. Locally advanced and surgically unresectable tumors are defined as those encasing the adjacent arteries (celiac axis, SMA, and common hepatic artery) or occluding the SMV, PV, or SMV-PV confluence.

At the University of Texas MD Anderson Cancer Center (Houston, TX, USA), patients with borderline resectable pancreatic cancer were considered those whose tumors demonstrated a short-segment encasement of the hepatic artery, which is amenable to resection and reconstruction, without evidence of tumor extension to the celiac axis; abutment of the SMA involving less than or equal to $180^{\circ}$ of the circumference of the artery, or short-segment occlusion of the SMV, PV, or SMV-PV confluence with an option for vascular reconstruction based on a normal SMV below and PV above the area of tumor involvement. ${ }^{12}$ The American HepatoPancreatico-Biliary Association (AHPBA) consensus conference on pancreatic cancer (2009) ${ }^{35}$ expanded the venous involvement criteria, allowing surgery even in cases of tumor abutment of the SMV-PV with or without impingement and narrowing of the lumen. The National Comprehensive Cancer Network later adopted some of these AHPBA guidelines in its most recent version (2014) and allows SMV-PV abutment without impingement and narrowing of the lumen. ${ }^{36-38}$ The criteria for arterial involvement (SMA and hepatic artery) are more clearly defined and do not show major differences.

The increasing advances of more aggressive surgery in pancreatic resections, together with advances and new promising applications in chemotherapy and radiotherapy, have led to the development of an important intermediate disease stage that is defined as borderline resectable disease. ${ }^{39-41}$ The definition of BRTs so far includes those tumors that exhibit the following: 1) no distant metastases;
2) venous involvement of the SMV or PV with distortion or narrowing of the vein or occlusion of the vein with suitable vessel proximal and distal, allowing for safe resection and replacement; 3 ) the encasement of the gastroduodenal artery up to the hepatic artery, with either short segment encasement or direct abutment of the hepatic artery, without extension to the celiac axis; and 4) tumor abutment of the SMA not to exceed greater than $180^{\circ}$ of the circumference of the vessel wall. It is evident that the detailed delineation of peripancreatic vascular involvement based on CT imaging is of great importance for accurate characterization of BRTs. ${ }^{42}$

Surgery is considered the mainstay of treatment for pancreatic cancer, with achievement of a complete resection being linked to best possible outcome. ${ }^{43-45}$ However, multimodality treatment which includes chemotherapy and radiotherapy is currently being extensively investigated, especially in the borderline resectable group of pancreatic cancer patients for the following two reasons: 1) it is necessary to achieve a margin-negative resection, and 2) neoadjuvant protocols will help identify those patients with better tumor biology who are more likely to benefit from surgery after neoadjuvant therapy. ${ }^{12}$

Regarding borderline resectable pancreatic cancer, while the tumor relationship to the arterial structures as seen on optimal CT imaging and based on the above criteria accurately predicts the arterial involvement, the same cannot be said of venous imaging. While tumor encasement of the SMV ( $>180^{\circ}$ contact with tumor) or venous occlusion is predictive of venous involvement, the same cannot be concluded about tumor abutment to the SMA (tumor-vessel contact $<180^{\circ}$ ).

$\mathrm{Lu}$ et $\mathrm{al}^{46}$ studied the vascular relationships of pancreatic adenocarcinomas on $\mathrm{CT}$ imaging and graded them on a $0-4$ scale. Grade 0 was designated if there was a plane between tumor and vessel, grade 1 when the tumor-vessel contact was less than or equal to $90^{\circ}$, grade 2 when the tumor-vessel contact was greater than $90^{\circ}$ up to $180^{\circ}$, grade 3 when the tumor-vessel contact exceeded $180^{\circ}$ but was less than $270^{\circ}$, and grade 4 when the tumor-vessel contact was greater than or equal to $270^{\circ}$. In their series, four of seven vessels with a grade 2 relation required vascular resection. However, in one patient in their study who was graded as 3, the tumor was easily separated from the SMV. Their data seemed to indicate that mere contiguity of tumor to vein does not signify invasion.

\section{Accuracy of MDCTA}

The role of the radiologist in a case of pancreatic cancer is accurate diagnosis, staging, and estimation of resectability, 
as this is defined by the specific described criteria concerning mainly vessel infiltration. MDCT has been used to evaluate resectability due to its high accuracy, and various grading systems have been proposed. It should be emphasized that only patients with $\mathrm{R} 0$ resections benefit from surgery, while if either microscopic (R1) or gross disease (R2) is left behind, little or no survival benefit is provided compared with palliative bypass surgery.

Kaneko et $\mathrm{al}^{47}$ retrospectively compared MDCTA done preoperatively in cases of pancreatic head cancers to the surgical outcomes. In this study, MDCTA was found to have a sensitivity, specificity, positive predictive value (PPV), negative predictive value (NPV), and accuracy of $100 \%, 71 \%, 85 \%, 100 \%$, and $89 \%$, respectively, which was similar to the results reported by Fusari et $\mathrm{al}^{26}$ and Zamboni et $a{ }^{48}$ Results vary according to the various generations of MDCT scanners used.

Shrikhande et $\mathrm{al}^{40}$ studied 12 patients with borderline resectable disease according to the MD Anderson Cancer Center classification. They correlated the MDCT results with the intraoperative and histopathology findings. Eight of the 12 patients finally underwent a curative R0 resection while another two had microscopically positive margins (R1 resections). They proposed a combined evaluation of three imaging parameters on MDCTA: the maximum degree of circumferential contact (CC), the length of contact (LC) of the tumor with major vessels, and the luminal narrowing of vessels at the point of contact with the tumor (venous deformity [VD]). This could be helpful to select patients with apparently borderline disease for potentially curative surgical resection. This grading system needs, however, to be validated in a larger number of patients suffering from pancreatic adenocarcinoma.

Similarly, Kent et $\mathrm{al}^{49}$ have proposed a CT grading system for the estimation of resectability in pancreaticobiliary tumors. They created a five-point scale that describes the imaging relationship of the pancreatobiliary mass to adjacent vessels, PV, SMV, SMA, and celiac trunk. An increasing grade is associated with a higher probability of unresectability or $\mathrm{R} 1$ resection. ${ }^{49}$

Brügel et $\mathrm{al}^{50}$ confirmed that thin-slice MPR obtained with multislice helical CT provided an exact depiction of the relation between the tumor and the potentially infiltrated vessels, so the assessment of local resectability is much improved.

To investigate the accuracy of MDCT in preoperatively determining the surgical resectability of pancreatic adenocarcinomas, Işcanlı et al $^{51}$ evaluated retrospectively MDCT, surgery, and pathological results of 274 patients with pancreatic adenocarcinoma. A total of 124 out of $274(56 \%)$ patients (83 males, mean age: 60 years) underwent laparoscopy and/or laparotomy. The surgery was not performed in 150 of 274 (54\%) patients who were not suitable for curative surgery according to the MDCT findings. The results of the abovementioned study showed that the sensitivity, specificity, PPV, NPV, and accuracy of MDCT in determining the surgical resectability rates of pancreatic adenocarcinomas were $100 \%, 72 \%, 78 \%, 100 \%$, and $86 \%$, respectively.

Olivié et $\mathrm{al}^{52}$ evaluated prospectively the ability of MDCT to predict resectability of pancreatic head cancer. Ninety-one patients (53 men, 38 women; mean age: 61 years) diagnosed with cancer of the head of the pancreas underwent a preoperative contrast enhanced triphasic 16-slice MDCT. Sixty-three were considered inoperable because of advanced local disease, metastatic disease, or high surgical risk. In reference to the study group (28 patients), they concluded that, when compared to surgical outcome, the PPV of MDCT for surgically resectable disease was $100 \%$ (23/23) and the NPV (prediction of unresectability) was also $100 \%(5 / 5)$. Accuracy was 100\% (28/28).

The sensitivity, specificity, PPV, NPV, and accuracy of MDCT and MDCTA of the various studies mentioned in this paper are displayed in Table 1.

Table I Results of MDCT and MDCTA studies

\begin{tabular}{|c|c|c|c|c|c|}
\hline MDCT-MDCTA & $\begin{array}{l}\text { Specificity } \\
\%\end{array}$ & $\begin{array}{l}\text { Sensitivity } \\
\%\end{array}$ & $\begin{array}{l}\text { PPV } \\
\%\end{array}$ & $\begin{array}{l}\text { NPV } \\
\%\end{array}$ & $\begin{array}{l}\text { Accuracy } \\
\%\end{array}$ \\
\hline Fusari et al, $2010^{26}$ & 100 & 92 & 100 & 78 & 94 \\
\hline DeWitt et al, $2004^{27}$ & 78 & 84 & & & 86 \\
\hline Imbriaco et al, $2005^{33}$ & 89 & 94 & & & \\
\hline Kaneko et al, $2010^{47}$ & 71 & 100 & 85 & 100 & 89 \\
\hline Zamboni et al, $2007^{48}$ & 72 & 100 & 89 & 100 & \\
\hline Brügel et al, $2004^{50}$ & 97 & 74 & 88 & & \\
\hline Grieser et al, $2010^{65}$ & 93 & 100 & & & \\
\hline Ișcanlı et al, 2014 $4^{51}$ & 72 & 100 & 78 & 100 & 86 \\
\hline Olivié et al, $2007^{52}$ & 100 & 100 & 100 & 100 & 100 \\
\hline
\end{tabular}

Abbreviations: MDCT, multidetector computed tomography; MDCTA, multidetector computed tomographic angiography; NPV, negative predictive value; PPV, positive predictive value. 


\section{Technical considerations}

It is of great importance to choose the timing of the contrast material bolus to maximize sensitivity for the detection and staging of pancreatic cancer. Currently, with 64-section $\mathrm{CT}$, multiple discrete phases of vascular and parenchymal enhancement can easily be achieved, but the radiologist should select what is most appropriate with regard to the clinical question and to minimize patient radiation exposure.

There are three methods, well described in the literature, to determine the scan timing: empiric timing, use of a test bolus, or bolus tracking. With the test bolus technique, multiple low-dose scans are acquired at a single level that is usually selected at the level of the celiac axis, following the administration of $20 \mathrm{~mL}$ of iodinated contrast material. A region of interest is positioned over the aorta, and a temporal graph of the contrast enhancement is obtained. The time of peak enhancement is then used to calculate the optimum timing for each phase of acquisition.

With the bolus tracking technique, low-dose continuous scans are obtained after the administration of 150-200 mL of contrast material with automatic power injectors at a rate of 4-6 mL/sec through an intravenous catheter usually placed in an antecubital vein. A region of interest of $40-50 \mathrm{~mm}^{2}$ and covering about $70 \%$ of the cross-sectional area of the aorta is positioned on the abdominal aorta at about the level of the celiac axis. Scanning is triggered when a predefined attenuation level (in Hounsfield units) is reached.

In pancreatic imaging, accurate bolus timing is critical. However, the use of a fixed empiric delay is unlikely to ensure the peak parenchymal and vessel enhancement in all patients, so this technique is not recommended.

We should keep in mind that maximal pancreatic parenchymal enhancement is influenced by many factors. These are technical factors such as the generation of CT scanner or contrast material-related factors including the rate of injection, the volume and concentration of iodine, and finally, patient-related factors such as age, weight, and cardiac output. This leads to a wide intraindividual variability, making peak parenchymal enhancement extremely important in test bolus or bolus tracking techniques. In our radiology department, we routinely use bolus tracking with automatic power injectors, and we set a region of interest on the abdominal aorta at the level of the celiac axis. The predefined enhancement threshold is set at $150 \mathrm{HU}$.

The triple phase taken includes a nonenhanced phase, a late arterial phase (10-second delay from the time of peak aortic enhancement), and a portal venous phase (35-second delay). Contrast-enhanced phases are performed craniocaudally, with thin collimation $(0.5$ or $0.625 \mathrm{~mm})$, $120 \mathrm{kVp}$, and automatic modulation of the milliamperes.
Five-millimeter axial images and $5 \times 5 \mathrm{~mm}$ coronal and sagittal reformatted images are sent to the picture archiving and communication system, and $0.5 \mathrm{~mm}$-thick sections are sent to workstations for postprocessing.

It has been shown in many studies that the late arterial phase of imaging (which corresponds to the pancreatic phase of parenchymal enhancement) ensures maximal conspicuity of hypovascular tumors. At the same time, there is also adequate arterial and mesenteric venous enhancement for the detection of possible vascular invasion. ${ }^{53,54} \mathrm{~A}$ pure arterial phase is not routinely performed as the late arterial one also provides excellent enhancement of the arterial system ${ }^{31,32}$ (Figure 1A and B). Only when MDCT arteriography should be performed, in cases of locally advanced disease, is an additional early arterial phase study performed. It is well known that the portal venous phase of imaging is the ideal phase for detecting liver metastases, but it can also be used to create reconstructed images of the venous structures (Figures 1C and 2C). A second look at the pancreas during this phase can occasionally be very useful, specifically in cases of isoattenuating tumors in the parenchymal phase. Then, according to the location of the tumor and the needs for surgery planning, a variety of postprocessing techniques might be used: MPR, maximum intensity projection (MIP), volume rendering (VR), and curved planar reformations on the course of vessels or the main pancreatic duct. Many reports have shown the added value of high-quality MPR images in the accurate evaluation of vascular invasion and resectability of pancreatic cancer. ${ }^{55,56}$

For the preoperative evaluation of pancreatic tumors, it is necessary to evaluate axial images; coronal, sagittal, and curved MPR images; and vascular, MIP, or VR reformatted images from data obtained during both the pancreatic parenchymal and portal venous phases. Reconstructions of curved MPR-MIP images of the hepatobiliary system are occasionally needed for pancreatic head cancer. Vascular reformatted images and thickslab oblique images can be prepared by experienced radiographers and viewed on the picture archiving and communication system by the radiologists. Additional image processing can also be performed according to specific needs. The surgeons may also need advanced postprocessing, including surgical simulation to be better prepared for the surgery.

In the mid-2000s, estimation of potential resectability was decided on the basis of the venous phase of SMA angiographic findings. With the advances of CT and CT angiography, interventional techniques such as digital angiography are no longer used for diagnostic purposes. MDCT axial source data combined with VR and curved MPR images represent the optimal method for the evaluation of pancreatic cancer resectability. ${ }^{56,57}$ As described in the previous section, there 

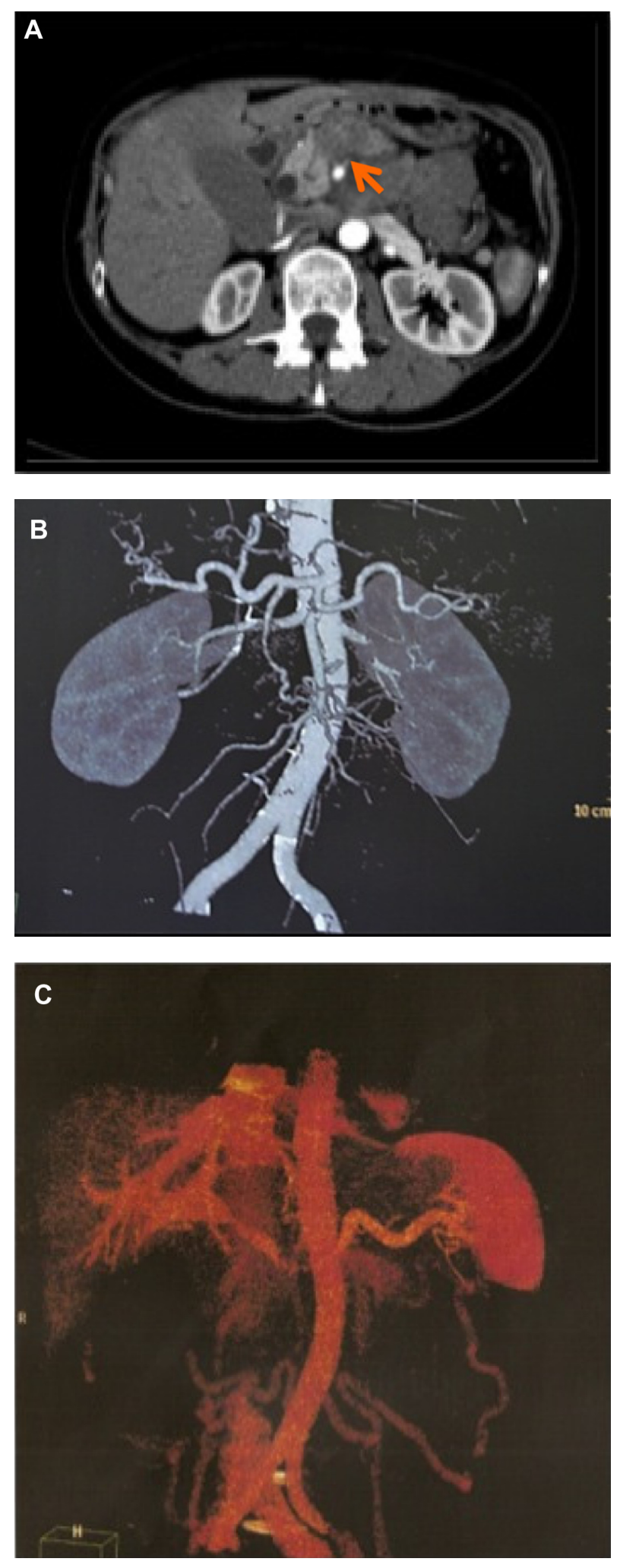

Figure I Evaluating the level of involvement of vessels.

Notes: (A) shows a 53-year-old patient with a pancreatic tumor in the isthmus (arrow); At computed tomographic angiography, the arterial phase of the examination revealed no evidence of infiltration of the branches of the abdominal aorta, celiac trunk, and superior mesenteric artery (B); however, the threedimensional reconstruction of splenoportal axis revealed two severe stenoses: one at the liver hilum and the other at the confluence of the portal and mesenteric vein, while many collateral vessels are also seen (C). are many CT criteria used to suggest potential unresectability, including a circumferentially narrowed or occluded SMV or PV; increasing degree of circumferential venous involvement by the tumor; the "teardrop" mesenteric vein sign; and the presence of small, dilated peripancreatic veins, indicating PV infiltration or thrombosis. ${ }^{58}$ The teardrop mesenteric vein sign was described by Hough et al, ${ }^{58}$ indicating tumor involvement of the SMV. It was supposed that a focally-tethered, teardrop-shaped SMV was related to desmoplastic reaction around the tumor, predicting the unresectability of adenocarcinoma of the pancreatic head. As surgical techniques have changed over the years, imaging criteria have also been modified, and this sign is no longer considered to represent a sign of unresectability.

Nevertheless, when assessing axial images, we can still use the grading system proposed by Raptopoulos et al. ${ }^{59} \mathrm{In}$ their system, five grades of possible vascular invasion were recognized as follows: grade 0 , normal, with a fat plane or normal pancreas between the tumor and vessel; grade 1, loss of the fat plane between the tumor and vessel, with or without displacement of the vessel; grade 2, flattening or slight irregularity of one side $\left(<180^{\circ}\right.$ of the perimeter) of the vessel, a finding that is associated with questionable venous involvement but still indicating a resectable tumor; grade 3, encased vessel with tumor extending around at least at two sides ( $>180^{\circ}$ of the perimeter), altering vessel contour and producing concentric or eccentric narrowing of the lumen (associated with definite tumor invasion, although not necessarily excluding surgery as long as en bloc venous resection is possible); and grade 4, at least one occluded major vessel (mostly unresectable, although again, a short-segment focal venous occlusion with superior and inferior patency may allow local resection). Individual assessment of all vessels, such as the PV, splenic vein, and SMV as well as all of the major branches (Figure 1), should be systematically made using a combination of axial and reformatted CT images as described earlier before making any surgical decision.

\section{Surgical challenges}

In recent years, there has been a tendency in pancreatic surgery toward performing en bloc venous resection of the SMV-PV confluence when this involvement is the only potential difficulty. ${ }^{60}$ There are few reports in the literature on arterial resections where there is such involvement, but still, SMA or celiac axis infiltration remains a contraindication for resection. This is based on the fact that the mesenteric neural plexus is frequently involved together with the arteries, so even if technically feasible, the resection would be probably ontologically insufficient. It should be emphasized that these en bloc vascular 
resections are performed only in an effort to achieve a negative resection margin in cases where $\mathrm{R} 0$ resection would otherwise be impossible due to locally advanced disease. There is evidence in the recent literature that segmental en bloc SMV-PV resection can be beneficial in selected patients with BRTs. ${ }^{61}$

Many studies have shown that CT can be used to predict the definite need for venous resection ${ }^{62}$ while collaboration and discussion with the surgeon is very important in these difficult and challenging cases. As multiple strategies and different techniques for revascularization exist, the surgeon should know details of the position of the venous structures such as the gastrocolic trunk, the inferior mesenteric vein, and the first jejunal branch as well as their respective distances from each other, the tumor, and the SMV-PV confluence. Only by providing volumetric data using the techniques of MDCTA can the surgery be correctly planned and the position of bypass grafts be selected.

It is generally important to pay particular attention to the relationship of the tumor to the SMV-PV confluence and the measure of the length of vascular involvement. End-to-end anastomosis cannot be performed if more than $2 \mathrm{~cm}$ of the SMV is removed, so in these cases, it is usually better to conserve the SMV-PV anastomosis and insert an interposition graft. The excision of the confluence and the splenic vein can lead to portal hypertension with potential upper gastrointestinal variceal bleeding. Despite this fact, in cases of SMV-PV confluence involvement, extensive resection with ligation of the splenic vein may be required.

Evaluation of arterial involvement is also of great importance for surgery planning and is based on a combination of axial source images and postprocess-rendered images (Figure 2). On axial images, a periarterial cuff of soft tissue due to tumor involvement can be directly visualized. It is also essential to recognize preoperatively any anatomic variation of the arteries that could interfere in the surgical area (eg, hepatic artery derived from SMA, etc).

\section{Critical points on imaging analysis}

It should be emphasized that MIP and VR images cannot depict a perivascular cuff of soft tissue due to neoplastic infiltration as long as there are no changes in the vessel's caliber. However, postprocessed images allow better anatomic delineation and assessment of the extent of involvement (Figure 3). Optimal results are, in any case, achieved when axial source images are combined with postprocessed images. ${ }^{59}$ Several grading systems ${ }^{59,63}$ can be used to quantify circumferential arterial encasement by tumor thus predicting the likelihood of successful resection.
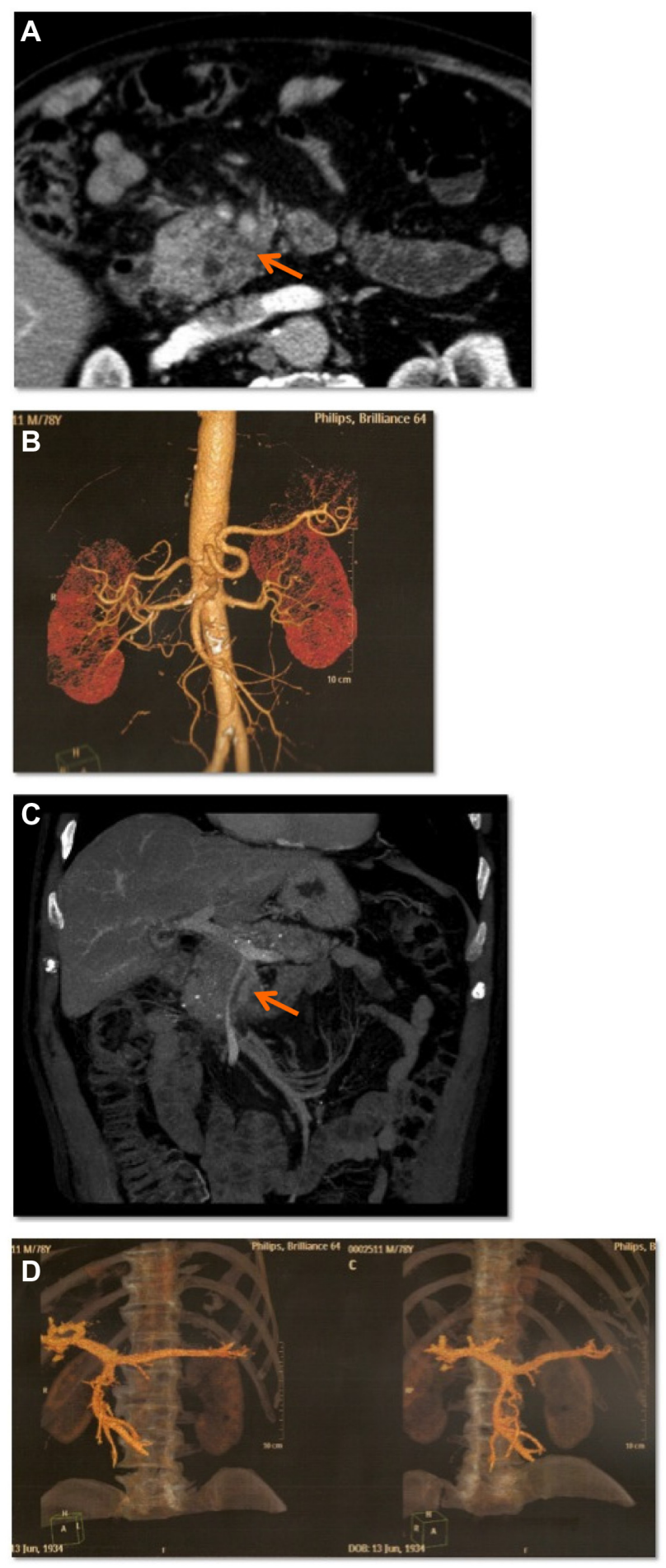

Figure $2 \mathrm{~A}$ combination of axial source images and postprocess-rendered images to evaluate arterial involvement.

Notes: (A) shows a 78-year-old patient with pancreatic cancer in the head and uncinate process (arrow); On computed tomographic angiography, no infiltration or narrowing of the celiac artery or the superior mesenteric artery and their branches was revealed (B); In the portal phase, the tumor was shown to have contact with the superior mesenteric vein on one side at its proximal portion (arrow), without thrombosis or marked stenosis (C); There was no change in the diameter of the portal and splenic veins, as shown on the three-dimensional volume rendering (D); The patient was brought to surgery because the tumor was characterized as "borderline resectable," where it was found that the superior mesenteric vein was infiltrated at a greater length, and there was also invasive expansion in the retroperitoneal tissues not depicted preoperatively by imaging. 

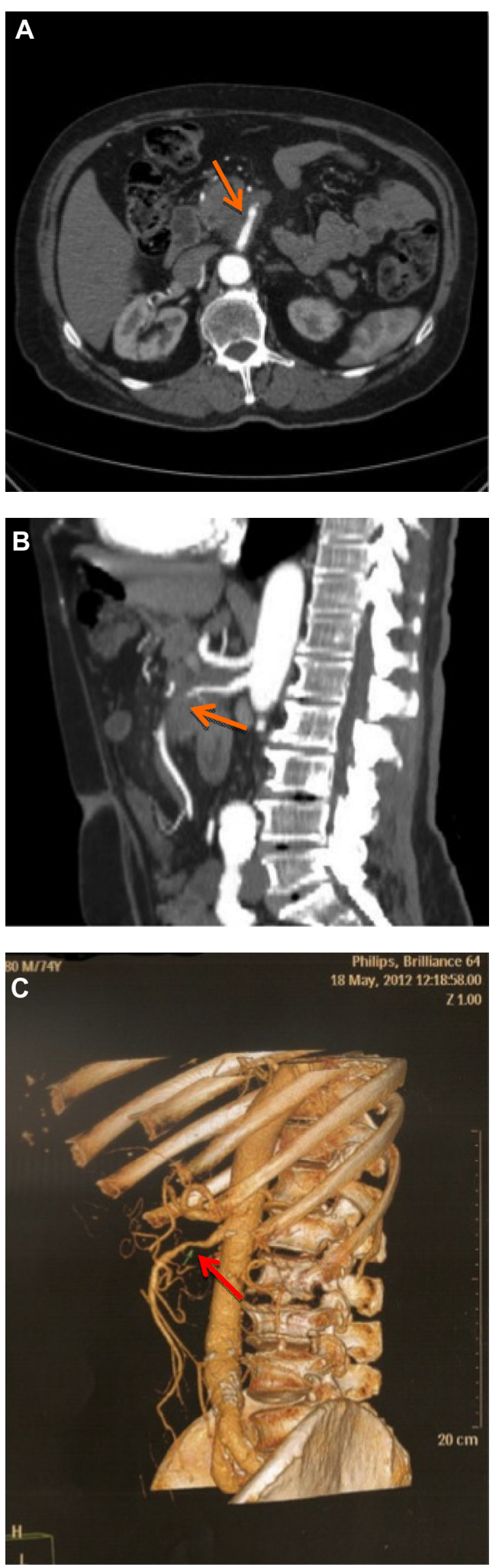

Figure 3 Postprocessed images allow better anatomic delineation and assessment of the extent of vascular involvement.

Notes: A pancreatic tumor in the head and uncinate process (arrow) is shown in transverse axial image (A); and (B) shows multiplanar reconstruction in a sagittal plane showing stenosis of the superior mesenteric artery close to the aorta (arrow), the tumor encases the artery at an angle greater than $180^{\circ}$; (C) shows a threedimensional reconstruction image from the same patient showing the length of superior mesenteric artery involvement (arrow).
In our institution, image analysis and interpretation is done using axial images as well as MPR. Curved planar reformations and VR are also used where deemed necessary for studying the relationship of the tumor to peripancreatic vessels. The parameters we evaluate are the tumor location and its relation with the relevant peripancreatic vessels.

Tumor relationship with the PV, SMV, and SMA is assessed using two parameters. The first requires measurement of the maximum degree of $\mathrm{CC}$ of the tumor with the vessel. This measurement is obtained using the angle measurement tool available on the PACS software (Philips Brilliance 64, Workspace-Philips Healthcare, Best, the Netherlands). The angle (criteria for maximum degree of CC) obtained is classified as follows: grade 0 , no contact of the tumor with the vessel; grade 1 , tumor to vessel contact $<90^{\circ}$; grade 2 , tumor to vessel contact $\geq 90^{\circ}$ and $<180^{\circ}$; grade 3, tumor to vessel contact $\geq 180^{\circ}$ but $<270^{\circ}$; and grade 4 , tumor to vessel contact $\geq 270^{\circ}$.

The second parameter is the LC of the tumor with the vessel. This is measured both on the coronal/sagittal reformations and the axial images.

The degree of VD is also measured. This represents the third parameter that is measured in both SMV and PV. The minimum luminal diameter of the vein in contact with the tumor is the numerator, and the denominator is the diameter of the vein immediately proximal and not in contact with the tumor. The criteria for VD are outlined below: grade 0, no luminal narrowing; grade 1, $<50 \%$ narrowing; and grade $2, \geq 50 \%$ narrowing. The tumor relationship with adjacent organs and structures is also recorded. Image analysis and recording includes the presence or absence of hepatic metastases, ascites, peritoneal nodules, and adenopathy.

\section{Publication-based evidence}

All available major publications (33 in total) from the years 2009 to 2014 which demonstrate that accurate pretreatment assessment of resectability is crucial to the design of appropriate preoperative protocols for the operating team were retrieved from PubMed/MEDLINE, Embase, and the Cochrane Central Register of Controlled Trials. Preoperative knowledge of the degree of vascular involvement will facilitate intraoperative conditions and minimize the potential complications of adding a vascular procedure to an already risky operation. MDCTA seems to be the technique of choice, permitting detailed evaluation of peripancreatic arteries and veins before therapeutic selection and design, specifically in borderline cases. $^{5,11-13,16,17,19,21,23,25-27,29,33,36-41,44,45,48-50,52,53,56,57,63-66}$ 


\section{Conclusion}

Pancreatic resections are difficult and demanding procedures, and every effort should be made to avoid adding a vascular resection, if possible, without compromising radicalism from the oncology point of view.

In conclusion, at present, it is possible to achieve a complete resection in selected patients who are found to have BRTs on preoperative imaging using dedicated MDCT and MDCTA protocols. Preoperative MDCTA-based grading systems can help identify such patients so that timely and effective treatment can be ensured.

\section{Disclosure}

The authors report no conflicts of interest in this work.

\section{References}

1. Sahmoun AE, D'Agostino RA, Bell RA, Schwenke DC. International variation in pancreatic cancer mortality for the period 1955-1998. Eur J Epidemiol. 2003;18(8):801-816.

2. Jemal A, Siegel R, Ward E, et al. Cancer statistics, 2006. CA Cancer J Clin. 2006;56(2):106-130.

3. Zamboni G, Capelli P, Pesci A, Beghelli S, Lüttges J, Klöppel G. Pancreatic head mass: what can be done? Classification: the pathological point of view. JOP. 2000;1(3 Suppl):77-84.

4. Cooperman AM, Kini S, Snady H, Bruckner H, Chamberlain RS. Current surgical therapy for carcinoma of the pancreas. J Clin Gastroenterol. 2000;31(2):107-113.

5. Wray CJ, Ahmad SA, Matthews JB, Lowy AM. Surgery for pancreatic cancer: recent controversies and current practice. Gastroenterology. 2005;128(6):1626-1641.

6. Birkmeyer JD, Siewers AE, Finlayson EV, et al. Hospital volume and surgical mortality in the United States. N Engl J Med. 2002;346(15): 1128-1137.

7. Takai S, Satoi S, Toyokawa H, et al. Clinicopathologic evaluation after resection for ductal adenocarcinoma of the pancreas: a retrospective, single-institution experience. Pancreas. 2003;26(3):243-249.

8. Neoptolemos JP, Stocken DD, Dunn JA, et al; European Study Group for Pancreatic Cancer. Influence of resection margins on survival for patients with pancreatic cancer treated by adjuvant chemoradiation and/or chemotherapy in the ESPAC-1 randomized controlled trial. Ann Surg. 2001;234(6):758-768.

9. Sohn TA, Yeo CJ, Cameron JL, et al. Resected adenocarcinoma of the pancreas-616 patients: results, outcomes, and prognostic indicators. J Gastrointest Surg. 2000;4(6):567-579.

10. Winter JM, Cameron JL, Campbell KA, et al. 1423 pancreaticoduodenectomies for pancreatic cancer: A single-institution experience. J Gastrointest Surg. 2006;10(9):1199-1210; discussion 1210-1211.

11. Faria SC, Tamm EP, Loyer EM, Szklaruk J, Choi H, Charnsangavej C. Diagnosis and staging of pancreatic tumors. Semin Roentgenol. 2004;39(3):397-411.

12. Varadhachary GR, Tamm EP, Abbruzzese JL, et al. Borderline resectable pancreatic cancer: definitions, management, and role of preoperative therapy. Ann Surg Oncol. 2006;13(8):1035-1046.

13. Brown KM, Siripurapu V, Davidson M, et al. Chemoradiation followed by chemotherapy before resection for borderline pancreatic adenocarcinoma. Am J Surg. 2008;195(3):318-321.

14. Tamm E, Charnsangavej C, Szklaruk J. Advanced 3-D imaging for the evaluation of pancreatic cancer with multidetector CT. Int J Gastrointest Cancer. 2001;30(1-2):65-71.

15. Pisters PW, Lee JE, Vauthey JN, Charnsangavej C, Evans DB. Laparoscopy in the staging of pancreatic cancer. Br J Surg. 2001;88(3):325-337.
16. Mayo SC, Austin DF, Sheppard BC, Mori M, Shipley DK, Billingsley KG. Evolving preoperative evaluation of patients with pancreatic cancer: does laparoscopy have a role in the current era? J Am Coll Surg. 2009;208(1):87-95.

17. Brennan DD, Zamboni GA, Raptopoulos VD, Kruskal JB. Comprehensive preoperative assessment of pancreatic adenocarcinoma with 64-section volumetric CT. Radiographics. 2007;27(6):1653-1666.

18. Arslan A, Buanes T, Geitung JT. Pancreatic carcinoma: MR, MR angiography and dynamic helical CT in the evaluation of vascular invasion. Eur J Radiol. 2001;38(2):151-159.

19. Grenacher L, Klauss M, Dukic L, et al. [Diagnosis and staging of pancreatic carcinoma: MRI versus multislice-CT - a prospective study]. Rofo. 2004;176(11):1624-1633. German.

20. Dickersin K, Scherer R, Lefebvre C. Identifying relevant studies for systematic reviews. BMJ. 1994;309(6964):1286-1291.

21. Khan I, Conlon K. Pancreatic adenocarcinoma. In: Garden O, editor Hepatobiliary and Pancreatic Surgery. 4th ed. Oxford: Elsevier, 2009: 283-298.

22. Bluemke DA, Cameron JL, Hruban RH, et al. Potentially resectable pancreatic adenocarcinoma: spiral CT assessment with surgical and pathologic correlation. Radiology. 1995;197(2):381-385.

23. Bronstein YL, Loyer EM, Kaur H, et al. Detection of small pancreatic tumors with multiphasic helical CT. AJR Am J Roentgenol. 2004;182(3): 619-623.

24. Diehl SJ, Lehmann KJ, Sadick M, Lachmann R, Georgi M. Pancreatic cancer: value of dual-phase helical CT in assessing resectability. Radiology. 1998;206(2):373-378.

25. Kitano M, Kudo M, Maekawa K, et al. Dynamic imaging of pancreatic diseases by contrast enhanced coded phase inversion harmonic ultrasonography. Gut. 2004;53(6):854-859.

26. Fusari M, Maurea S, Imbriaco M, et al. Comparison between multislice CT and MR imaging in the diagnostic evaluation of patients with pancreatic masses. Radiol Med. 2010;115(3):453-466.

27. DeWitt J, Devereaux B, Chriswell M, et al. Comparison of endoscopic ultrasonography and multidetector computed tomography for detecting and staging pancreatic cancer. Ann Intern Med. 2004;141(10): 753-763.

28. Legmann P, Vignaux O, Dousset B, et al. Pancreatic tumors: comparison of dual-phase helical CT and endoscopic sonography. AJR Am J Roentgenol. 1998;170(5):1315-1322.

29. Agarwal B, Abu-Hamda E, Molke KL, Correa AM, Ho L. Endoscopic ultrasound-guided fine needle aspiration and multidetector spiral CT in the diagnosis of pancreatic cancer. Am J Gastroenterol. 2004;99(5): $844-850$.

30. Graf O, Boland GW, Warshaw AL, Fernandez-del-Castillo C, Hahn PF, Mueller PR. Arterial versus portal venous helical CT for revealing pancreatic adenocarcinoma: conspicuity of tumor and critical vascular anatomy. AJR Am J Roentgenol. 1997;169(1):119-123.

31. McNulty NJ, Francis IR, Platt JF, Cohan RH, Korobkin M, Gebremariam A. Multi - detector row helical CT of the pancreas: effect of contrast-enhanced multiphasic imaging on enhancement of the pancreas, peripancreatic vasculature, and pancreatic adenocarcinoma. Radiology. 2001;220(1):97-102.

32. Fletcher JG, Wiersema MJ, Farrell MA, et al. Pancreatic malignancy: value of arterial, pancreatic, and hepatic phase imaging with multidetector row CT. Radiology. 2003;229(1):81-90.

33. Imbriaco M, Megibow AJ, Ragozzino A, et al. Value of the singlephase technique in MDCT assessment of pancreatic tumors. AJR Am J Roentgenol. 2005;184(4):1111-1117.

34. Edge SB, Byrd DR, Compton CC, Fritz AG, Greene FL, Trotti A III, editors. AJCC Cancer Staging Manual. 7th ed. New York: SpringerVerlag; 2010.

35. National Comprehensive Cancer Network. NCCN practice guidelines for pancreatic cancer, Version 2 [webpage on the internet]. Fort Washington, PA: National Comprehensive Cancer Network; 2014. Available from: http://www.nccn.org/professionals/physician_gls/ recently_updated.asp. 
36. Vauthey JN, Dixon E. AHPBA/SSO/SSAT Consensus Conference on Resectable and Borderline Resectable Pancreatic Cancer: rationale and overview of the conference. Ann Surg Oncol. 2009;16(7):1725-1726.

37. Abrams RA, Lowy AM, O'Reilly EM, Wolff RA, Picozzi VJ, Pisters PW. Combined modality treatment of resectable and borderline resectable pancreas cancer: expert consensus statement. Ann Surg Oncol. 2009;16(7):1751-1756.

38. Callery MP, Chang KJ, Fishman EK, Talamonti MS, William Traverso L, Linehan DC. Pretreatment assessment of resectable and borderline resectable pancreatic cancer: expert consensus statement. Ann Surg Oncol. 2009;16(7):1727-1733.

39. Katz MH, Pisters PW, Evans DB, et al. Borderline resectable pancreatic cancer: the importance of this emerging stage of disease. $J$ Am Coll Surg. 2008;206(5):833-846; discussion 846-848.

40. Shrikhande SV, Arya S, Barreto SG, et al. Borderline resectable pancreatic tumors: is there a need for further refinement of this stage? Hepatobiliary Pancreat Dis Int. 2011;10(3):319-324.

41. Varadhachary GR, Tamm EP, Crane C, Evans DB, Wolff RA. Borderline resectable pancreatic cancer. Curr Treat Options Gastroenterol. 2005;8(5):377-384.

42. Bockhorn M, Uzunoglu FG, Adham M, et al; International Study Group of Pancreatic Surgery. Borderline resectable pancreatic cancer: a consensus statement by the International Study Group of Pancreatic Surgery (ISGPS). Surgery. 2014;155(6):977-988.

43. Campbell F, Smith RA, Whelan P, et al. Classification of R1 resections for pancreatic cancer: the prognostic relevance of tumour involvement within $1 \mathrm{~mm}$ of a resection margin. Histopathology. 2009;55(3): 277-283.

44. Kato K, Yamada S, Sugimoto H, et al. Prognostic factors for survival after extended pancreatectomy for pancreatic head cancer: influence of resection margin status on survival. Pancreas. 2009;38(6):605-612.

45. Shimada K, Sakamoto Y, Nara S, Esaki M, Kosuge T, Hiraoka N. Analysis of 5-year survivors after a macroscopic curative pancreatectomy for invasive ductal adenocarcinoma. World J Surg. 2010;34(8): 1908-1915.

46. Lu DS, Reber HA, Krasny RM, Kadell BM, Sayre J. Local staging of pancreatic cancer: criteria for unresectability of major vessels as revealed by pancreatic-phase, thin-section helical CT. AJR Am J Roentgenol. 1997;168(6):1439-1443.

47. Kaneko OF, Lee DM, Wong J, et al. Performance of multidetector computed tomographic angiography in determining surgical resectability of pancreatic head adenocarcinoma. J Comput Assist Tomogr. 2010;34(5):732-738.

48. Zamboni GA, Kruskal JB, Vollmer CM, Baptista J, Callery MP, Raptopoulos VD. Pancreatic adenocarcinoma: value of multidetector CT angiography in preoperative evaluation. Radiology. 2007;245(3): 770-778.

49. Kent TS, Raptopoulos V, Callery MP, Gautam S, Vollmer CM. Escalating computed tomography angiogram (CTA) grade predicts unresectability and margin status for pancreaticobiliary neoplasms. HPB (Oxford). 2010;12(2):115-122.

50. Brügel M, Rummeny EJ, Dobritz M. Vascular invasion in pancreatic cancer: value of multislice helical CT. Abdom Imaging. 2004;29(2): 239-245.
51. Işcanlı E, Türkvatan A, Bostancı EB, Sakaoğulları Z. Assessment of surgical resectability of pancreatic adenocarcinomas with multidetector computed tomography: what are the possibilities and problems? Turk $J$ Gastroenterol. 2014;25(4):416-423.

52. Olivié D, Lepanto L, Billiard JS, Audet P, Lavallée JM. Predicting resectability of pancreatic head cancer with multi-detector CT. Surgical and pathologic correlation. JOP. 2007;8(6):753-758.

53. Lu DS, Vedantham S, Krasny RM, Kadell B, Berger WL, Reber HA. Two-phase helical CT for pancreatic tumors: pancreatic versus hepatic phase enhancement of tumor, pancreas, and vascular structures. Radiology. 1996;199(3):697-701.

54. Boland GW, O'Malley ME, Saez M, Fernandez-del-Castillo C, Warshaw AL, Mueller PR. Pancreatic-phase versus portal vein-phase helical CT of the pancreas: optimal temporal window for evaluation of pancreatic adenocarcinoma. AJR Am J Roentgenol. 1999;172(3): 605-608.

55. Catalano C, Laghi A, Fraioli F, et al. Pancreatic carcinoma: the role of high-resolution multislice spiral CT in the diagnosis and assessment of resectability. Eur Radiol. 2003;13(1):149-156.

56. Brügel M, Link TM, Rummeny EJ, Lange P, Theisen J, Dobritz M. Assessment of vascular invasion in pancreatic head cancer with multislice spiral CT: value of multiplanar reconstructions. Eur Radiol. 2004;14(7):1188-1195.

57. Vargas R, Nino-Murcia M, Trueblood W, Jeffrey RB. MDCT in Pancreatic adenocarcinoma: prediction of vascular invasion and resectability using a multiphasic technique with curved planar reformations. AJR Am J Roentgenol. 2004;182(2):419-425.

58. Hough TJ, Raptopoulos V, Siewert B, Matthews JB. Teardrop superior mesenteric vein: CT sign for unresectable carcinoma of the pancreas. AJR Am J Roentgenol. 1999;173(6):1509-1512.

59. Raptopoulos V, Steer ML, Sheiman RG, Vrachliotis TG, Gougoutas CA, Movson JS. The use of helical CT and CT angiography to predict vascular involvement from pancreatic cancer: correlation with findings at surgery. AJR Am J Roentgenol. 1997;168(4):971-977.

60. Fuhrman GM, Leach SD, Staley CA, et al. Rationale for en bloc vein resection in the treatment of pancreatic adenocarcinoma adherent to the superior mesenteric-portal vein confluence. Pancreatic Tumor Study Group. Ann Surg. 1996;223(2):154-162.

61. Bachellier P, Nakano H, Oussoultzoglou PD, et al. Is pancreaticoduodenectomy with mesentericoportal venous resection safe and worthwhile? Am J Surg. 2001;182(2):120-129.

62. Bold RJ, Charnsangavej C, Cleary KR, et al. Major vascular resection as part of pancreaticoduodenectomy for cancer: radiologic, intraoperative, and pathologic analysis. J Gastrointest Surg. 1999;3(3): 233-243.

63. Shukla PJ, Barreto SG, Kulkarni A, Nagarajan G, Fingerhut A. Vascular anomalies encountered during pancreatoduodenectomy: do they influence outcomes? Ann Surg Oncol. 2010;17(1):186-193.

64. Talamonti M. Borderline resectable pancreatic cancer: a new classification for an old challenge. Ann Surg Oncol. 2006;13(8):1019-1020.

65. Grieser C, Steffen IG, Grajewski L, et al. Preoperative multidetector row computed tomography for evaluation and assessment of resection criteria in patients with pancreatic masses. Acta Radiol. 2010;51(10): 1067-1077.

66. Denecke T, Grieser C, Neuhaus P, Bahra M. Radiologic resectability assessment in pancreatic cancer. Rofo. 2014;186(1):23-29.

Reports in Medical Imaging

\section{Publish your work in this journal}

Reports in Medical Imaging is an international, peer-reviewed, open access journal publishing original research, reports, reviews and commentaries on all areas of medical imaging. The manuscript management system is completely online and includes a very quick and fair peer-review system, which is all easy to use. Visit http://www.dovep
from published authors

Dovepress

Visit http://www.dovepress.com/testimonials.php to read real quotes 